OPEN ACCESS

Edited by:

Karl Ljungberg,

Eurocine Vaccines $A B$, Sweden

Reviewed by:

Fok Moon Lum,

Stanford University, United States

Pierre Roques,

CEA Saclay, France

*Correspondence:

Wenije Tan

tanwj28@163.com

Specialty section:

This article was submitted to Vaccines and Molecular Therapeutics,

a section of the journal

Frontiers in Immunology

Received: 19 January 2021 Accepted: 09 March 2021

Published: 24 March 2021

Citation:

Zhao Z, Deng Y, Niu P, Song J, Wang $W$, Du Y, Huang B, Wang $W$, Zhang L, Zhao P and Tan W (2021)

Co-Immunization With CHIKV VLP and DNA Vaccines Induces a Promising Humoral Response in Mice.

Front. Immunol. 12:655743. doi: 10.3389/fimmu.2021.655743

\section{Co-Immunization With CHIKV VLP and DNA Vaccines Induces a Promising Humoral Response in Mice}

\author{
Zhimin Zhao ${ }^{1}$, Yao Deng ${ }^{1}$, Peihua Niu ${ }^{1}$, Jingdong Song ${ }^{1}$, Wen Wang ${ }^{1}$, Yongping $\mathrm{Du}^{2}$, \\ Baoying Huang ${ }^{1}$, Wenling Wang ${ }^{1}$, Leiliang Zhang ${ }^{3}$, Ping Zhao ${ }^{4}$ and Wenjie Tan ${ }^{1 *}$ \\ ${ }^{1}$ National Health Commission (NHC) Key Laboratory of Medical Virology, National Institute for Viral Disease Control and \\ Prevention, China CDC, Beijing, China, ${ }^{2}$ Key Laboratory of Laboratory Medicine, Ministry of Education, School of Laboratory \\ Medicine and Life Sciences, Wenzhou Medical University, Zhejiang, China, ${ }^{3}$ Institute of Basic Medicine, Shandong First \\ Medical University \& Shandong Academy of Medical Sciences, Jinan, China, ${ }^{4}$ Shanghai Key Laboratory of Biomedical \\ Protection, Department of Biomedical Protection, Faculty of Naval Medicine, Navy Medical University, Shanghai, China
}

Chikungunya fever is an acute infectious disease that is mediated by the mosquitotransmitted chikungunya virus (CHIKV), for which no licensed vaccines are currently available. Here, we explored several immunization protocols and investigated their immunity and protective effects in mice, with DNA- and virus-like particle (VLP)vaccines, both alone and in combination. Both DNA and VLP vaccine candidates were developed and characterized, which express CHIKV structural genes (C-E3-E2-6K-E1). Mice were immunized twice, with different protocols, followed by immunological detection and CHIKV Ross challenge. The highest antigen-specific lgG and neutralizing activity were induced by DNA and VLP co-immunization, while the highest cellular immunity was induced by DNA vaccination alone. Although all vaccine groups could protect mice from lethal CHIKV challenge, demonstrated as reduced viral load in various tissues, without weight loss, mice co-immunized with DNA and VLP exhibited the mildest histopathological changes and lowest International Harmonization of Nomenclature and Diagnostic Criteria (INHAND) scores, in comparison to mice with either DNA or VLP vaccination alone. We concluded that co-immunization with DNA and VLP is a promising strategy to inducing better protective immunity against CHIKV infection.

Keywords: immunity, virus-like particle, DNA vaccine, chikungunya virus, co-immunization

\section{INTRODUCTION}

Chikungunya fever (CHIKF) is a recurrent infectious disease caused by the chikungunya virus (CHIKV), which belongs to the genus Alphavirus of the Togaviridae family $(1,2)$. First documented in Tanzania in 1952, this virus has caused several CHIKF outbreaks, mainly in Africa and Asia. Widespread outbreak of CHIKF in recent years has made it a global public health problem (3). 
CHIKV is a positive-stranded RNA virus that encodes six structural (C-E3-E2-6K/TF-E1) and four non-structural (nsP1, helicase nsP2, nsP3, and polymerase nsP4) proteins (4). The two alphavirus envelope glycoproteins E1 and E2, each containing a single transmembrane domain, are responsible for mediating viral attachment (E2) and membrane fusion (E1) $(5,6)$. The prefusion E1/E2 heterodimer is arranged in 80 trimeric spikes, resulting in a viral particle with an icosahedral structure $(7,8)$.

Currently, there are no licensed CHIKV vaccines available for use; however, potential vaccine candidates are classified into seven types: inactivated vaccines, subunit vaccines, live-attenuated vaccines, recombinant virus-vectored vaccines, virus-like particle vaccines, chimeric vaccines, and nucleic acid vaccines $(9,10)$. Virus-like particles (VLPs) are generated by expression of the CHIKV structural cassette from a DNA expression plasmid transfected into human cells. The expressed structural proteins form particles that are indistinguishable from intact virions, but are replication-incompetent because they lack genomic viral RNA. Recently, a VLP vaccine, expressing by the measles virus vector, entered phase 2 clinical trials $(11,12)$. While this vaccine has demonstrated good safety and tolerability, it must be administered at least twice to reach $100 \%$ seroconversion and induce the production of sufficient levels of neutralizing antibodies. Importantly, an antibody-dependent enhanced infection (ADE) was reported during the infection process of CHIKV (13), and the severity of CHIKV infection increased in the presence of a subdominant immune response after immunization in mice (14, 15). Therefore, the impact of $\mathrm{ADE}$ on vaccination should also be considered during vaccine design.

Previous studies have shown that both CHIKV VLP and DNA vaccines can elicit specific immunity and protect mice from a lethal CHIKV threat $(16,17)$. Recently, a study demonstrated that co-immunization with different vaccine candidates could improve adaptive immunity (18). Coimmunization with both West Nile DNA and inactivated vaccines provide a synergistic increase in immunogenicity of mice (19). In addition, co-immunization with virus-like particles and DNA vaccines induce protection against respiratory syncytial virus infection and bronchiolitis (20). To date, a limited number of studies have investigated the efficacy of CHIKV vaccines, specifically the development of immunity by using DNA and VLP vaccines alone, or in combination. This study provides a comprehensive analysis of the immunogenicity and efficacy of CHIKV DNA and VLP vaccines, administered both individually and in combination, in mice.

\section{METHODS}

\section{Cell Lines and Viruses}

African green monkey Vero cells and human embryonic kidney 293 T cells were grown in Dulbecco's modified Eagle's medium (DMEM) (Hyclone, South Logan, UT, USA), supplemented with $10 \%$ fetal bovine serum (FBS) (Gibco, NY, USA) and $1 \%$ penicillin-streptomycin (Gibco, NY, USA). The live attenuated vaccine, CHIKV 181/clone25 strain (Asian), was obtained from
Terence Dermody through Add gene (pSinRep5-181/25ic, plasmid 60078) $(21,22)$. The CHIKV Ross infectious clone was provided by Dr. Ping Zhao (23). CHIKV 181/clone25 and CHIKV Ross infectious clone viruses were propagated and tittered in Vero cells.

\section{Construction of DNA-Based CHIKV Vaccine}

The full-length gene encoding CHIKV structural poly protein $(\mathrm{C}$ E3-E2-6K-E1) was amplified by polymerase chain reaction from CHIKV 181/25 with the following primers: CHIKV-forw 5'ATCGCCACCATGGAGTTTATCCCAACCC-3' and CHIKVrev 5'-CGGGATCCTTAGTGCCTGCTAAACGAC-3'. The products of the CHIKV structural genes were digested with EcoRV and BamHI, and cloned into the expression plasmid VRC-8301, under the control of the cytomegalovirus (CMV) immediate-early gene promoter (provided by Dr. Gary Nabel, $\mathrm{NIH}$ ) (24). The final expression construct was named pVRCCHIKV (DNA-based CHIKV vaccine) and screened using PCR and double restriction enzyme digestion. It was confirmed via sequencing in both directions to ensure fidelity (Figure 1A).

\section{Indirect Immunofluorescence Assay}

Briefly, 293T cells were transfected with pVRC-CHIKV and the VRC-8301 empty vector (mock)using PEI (Polyethylenimine) for $24 \mathrm{~h}$, followed by fixing with pre-cooled $4 \%$ paraformaldehyde, mobilizing in $0.2 \%$ Triton X-100, and blocking in $10 \%$ goat serum in PBS. Subsequently, the cells were incubated with rabbit polyclonal antibodies (Alpha Diagnostic Intl Inc, USA) against the CHIKV Capsid and E2 proteins, at $37^{\circ} \mathrm{C}$ for $2 \mathrm{~h}$. After washing with PBS, the cells were stained with secondary antibodies (FITC-labeled goat anti-rabbit IgG) and $0.1 \%$ DAPI at $37^{\circ} \mathrm{C}$ for $1 \mathrm{~h}$. Images of cells were acquired using a Leica TCS SP8 confocal microscope with LAS software (Leica Biosystems, Wetzlar, Germany)

\section{Preparation and Purification of CHIK VLPs}

For the preparation of CHIK VLPs, sub-confluent 293T cell cultures in $225 \mathrm{~cm}^{2}$ tissue culture flasks were transfected with $45 \mu \mathrm{g}$ of pVRC-CHIKV by using PEI. At $72 \mathrm{~h}$ post transfection, the culture media was collected, centrifuged at 4,000 rpm for $30 \mathrm{~min}$ at $4^{\circ} \mathrm{C}$, and filtered through a $0.22 \mu \mathrm{m}$ filter to remove cell debris. The VLPs were pelleted through a $20 \%$ sucrose cushion at $24,000 \mathrm{rpm}$ for $4 \mathrm{~h}$ at $4^{\circ} \mathrm{C}$, using a Beckman SW32 rotor. Pellets were re-suspended in TNE buffer containing $100 \mathrm{mM} \mathrm{NaCl}, 50$ $\mathrm{mM}$ Tris- $\mathrm{HCl}, \mathrm{pH} 7.2,1$ mM EDTA (Sigma, USA) and loaded on a discontinuous $60 \%$ and $20 \%$ sucrose gradient in TNE. Sucrose gradients were centrifuged at $24,000 \mathrm{rpm}$ for $4 \mathrm{~h}$ at $4^{\circ} \mathrm{C}$ using a Beckman SW41 rotor. The VLPs at the interface of $20-60 \%$ sucrose were collected, diluted, and pelleted through a $20 \%$ sucrose cushion at $24,000 \mathrm{rpm}$ for $4 \mathrm{~h}$ at $4^{\circ} \mathrm{C}$. The pellets were suspended in TNE buffer and stored at $-80^{\circ} \mathrm{C}$, until further use. The VLPs were analyzed by SDS-PAGE for the presence of CHIKV structural polyprotein (Figure 2). VLPs were quantified based on specific E2 protein content, which was determined by SDS-PAGE and calculated using ImageJ software. 


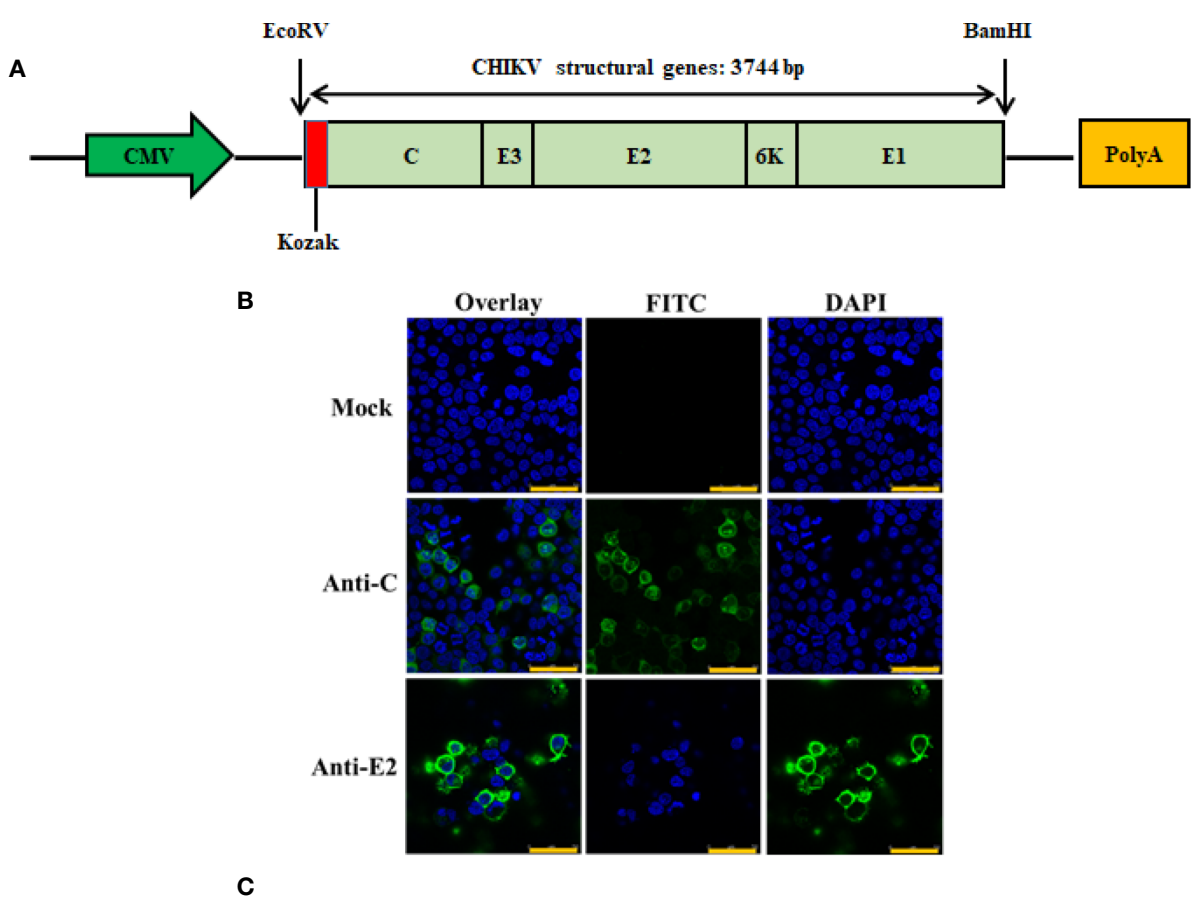

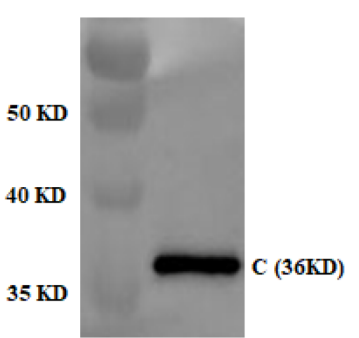

(a)

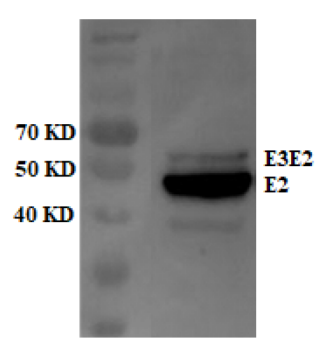

(b)

FIGURE 1 | Construction and antigen expression analysis of recombinant DNA-based CHIKV vaccine candidate. Schematic diagrams of the recombinant DNAbased vaccine encoding CHIKV structural genes. The flanking enzyme sites used for cloning, Kozak expression element and CHIKV structural genes (C-E3-E2-6KE1) are indicated and were cloned into the VRC-8301 vector (A). Immunofluorescence staining images showing the $293 T$ cells transiently transfected with the pVRCCHIKV or VRC-8301 (mock) at 24 h post-transfection. The capsid and E2 protein (FITC) and cell nuclei (DAPI) were stained in green and blue, respectively. Scale bars, $50 \mu \mathrm{m}$ (B). Expression of pVRC-CHIKV constructs was confirmed in vitro using CHIKV Capsid and E2 rabbit polyclonal antibodies for the Western blot of CHIKV Capsid (left) and E2 (right) proteins expressed in 293T cells by Western blotting (C).

\section{Transmission Electron Microscopy}

The purified CHIK-VLPs were negatively stained with $1 \%$ phosphotungstic acid (Ph 6.8). Briefly, $5 \mu \mathrm{L}$ of the VLPs were placed on a carbon-Formvar coated copper grid for $1 \mathrm{~min}$. Then grid was washed thrice in sterile triple-distilled water by floating the grid on water droplets for $45 \mathrm{~s}$, to remove excess sample and sucrose. Finally, the samples were stained with PTA solution for $1 \mathrm{~min}$. The air-dried grid was examined using a Tecnail2 transmission electron microscope (FEI, Eindhoven, Netherlands) at $120 \mathrm{kV}$ and recorded with a CCD camera.

\section{Immunizations and Challenge}

Female C57BL/6 mice, aged 6 to 7 weeks, were purchased from the Beijing Vital River Laboratory Animal Technology. All experiments were approved by the Committee on the Ethics of
Animal Experiments of the Chinese Center for Disease Control and Prevention (China CDC).

Mice were immunized (Figure 3) with DNA alone or coimmunized with DNA and VLP (hereafter DNA\&VLP), on day 0 and day 21, via intramuscular injection plus electroporation (i.m.+Ep), and with VLP alone or the mock vector, by subcutaneous injection. Samples were taken for immunological detection on days 14 and 35, post-vaccination.

Mice were challenged with $1.7 \times 10^{7} \mathrm{TCID}_{50}$ of the CHIKV Ross, which belongs to the East Central South Africa lineage (ECSA), in a total volume of $50 \mu \mathrm{L}$ by intranasal infection. The body weight and survival rate of mice were monitored daily. Animals were sacrificed either 14 days post-infection, or earlier if weight loss of more than $20 \%$ was observed. The heart, liver, spleen, lung, kidney, brain, and hind limbs of mice were harvested after sacrifice ( 3 mice per group). Half of the tissues 
A

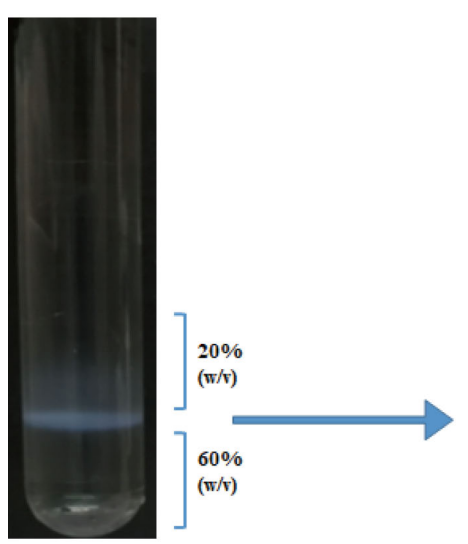

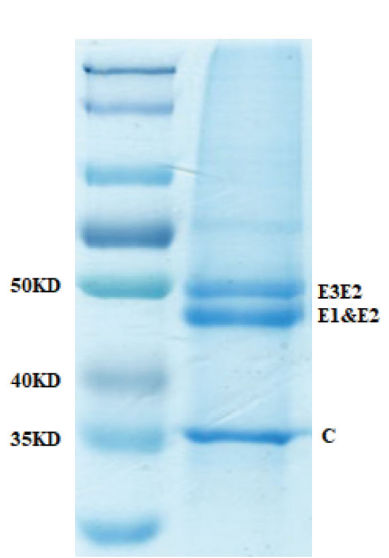

B

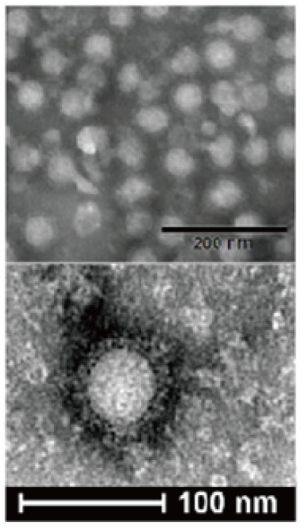

FIGURE 2 | Purification and characterization of CHIK-VLPS. CHIK-VLPs separated at the 20-60\% sucrose density gradient interface, SDS-PAGE followed by Coomassie blue staining (A). Electron microscopy of negatively stained CHIK-VLPs purified by sucrose gradient (B).

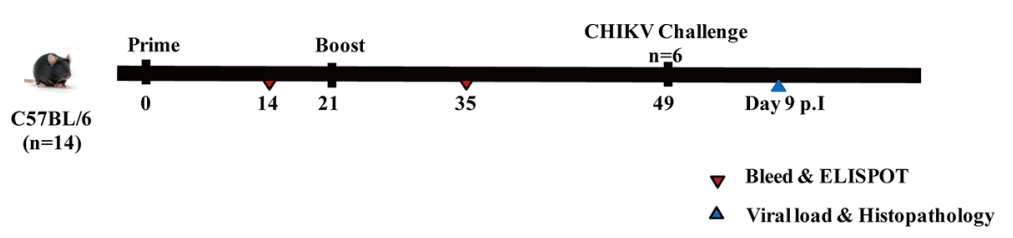

\begin{tabular}{ccccc}
\hline & Vaccine group & Prime & Boost & Root/Dose \\
\hline 1 & DNA & DNA & DNA & im. + EP, $20 \mu \mathrm{g} / 50 \mu \mathrm{L}$ \\
2 & VLP & VLP & VLP & s.c., $9 \mu \mathrm{g} / 50 \mu \mathrm{L}$ \\
3 & DNA+VLP & DNA+VLP & DNA+VLP & i.m. + EP $(20 \mu \mathrm{g}$ DNA+9 $\mu \mathrm{g}$ VLP $) / 50 \mu \mathrm{L}$ \\
4 & Mock & Mock & Mock & s.c. , 50 $\mu \mathrm{LPBS}$ \\
\hline
\end{tabular}

FIGURE 3 | Immunization and challenging schema of CHIKV vaccines. Mice ( $\mathrm{n}=14$ per group) were either mock immunized with PBS or vaccinated with DNA, VLP or DNA+VLP. Blood $(n=10)$ was collected for humoral immune response detection at day 14 and 35 by ELISA and plaque reduction neutralization assays at day 35.14 days after the prime and boost immunization, mice $(n=4)$ were sacrificed and cellular immune responses were analyzed in splenocytes. Mice ( $n=6)$ were challenged with $1.7 \times 10^{7} \mathrm{TCID}_{50}$ of the CHIKV Ross by intranasal infection at day 49. Body weight, survival rate, tissue viral load, and histopathological changes were evaluated.

were used for the determination of viral load, and the other half were fixed in a $4 \%$ formalin solution and sent to Beijing Zhongkewanbang Biotechnology Co., Ltd., for the preparation of hematoxylin and eosin (H\&E) stained sections, and pathological evaluation.

\section{Enzyme-Linked Immunosorbent Assay}

96-well plates (Thermo Fisher Scientific, USA) were coated with E1 (50 ng/well), E2 (50 ng/well), and VLPs (100 ng/well), followed by blocking with $200 \mu \mathrm{L}$ of $10 \%$ goat serum in PBS at $37^{\circ} \mathrm{C}$ for $2 \mathrm{~h}$. The plates were washed five times with PBST, followed by incubation with serially diluted post-vaccinated sera, including mock sera, at $37^{\circ} \mathrm{C}$ for $1 \mathrm{~h}$, followed by five washes with PBST. HRP-labeled goat anti-mouse IgG [1:5000], IgG1 [1:1500], and IgG2c [1:1500] antibodies were added at $37^{\circ} \mathrm{C}$ for $1 \mathrm{~h}$. After washing (as described above), $100 \mu \mathrm{L}$ of TMB was added to each well, incubated for $2 \mathrm{~min}$, and then quenched with $50 \mu \mathrm{L}$ of $2 \mathrm{M}$ $\mathrm{H}_{2} \mathrm{SO}_{4}$. Optical density values were measured using SPECTR Ostar Nano (BIO-GENE, China) at a wavelength of $450 \mathrm{~nm}$. The IgG2c/IgG1 ratios were calculated to define the $\mathrm{T}$ cell phenotype induced by vaccination since IgG2c and IgG1 levels are indicative of Th1 and Th2 responses, respectively (17).

\section{Neutralizing Antibodies}

Sera were heat-inactivated for $30 \mathrm{~min}$ at $56^{\circ} \mathrm{C}$, diluted 2 -fold from a starting dilution of 1:40, and mixed with an equal volume (10-15 pfu/well) of CHIKV Ross for $1 \mathrm{~h}$ at $37^{\circ} \mathrm{C}$. Virus dilutions without sera were prepared as controls. After $1 \mathrm{~h}$ of incubation, virus dilutions were applied to Vero cell monolayers for $1 \mathrm{~h}$ at $37^{\circ} \mathrm{C}$, and then overlaid with $4 \%$ methylcellulose. Plates were incubated for 3 days at $37^{\circ} \mathrm{C}$ and $5 \% \mathrm{CO}_{2}$. The overlay was then removed, and monolayers were stained with $1 \%$ crystal violet. 
Plaques were counted, and the neutralization titer was defined as the highest dilution resulting in a $50 \%$ reduction in plaque number $(24,25)$.

\section{IFN- $\gamma$ ELISpot Assay}

ELISpot assays were performed as previously reported (26). Briefly, 96-well ELISpot plates were coated with a purified antimouse IFN- $\boldsymbol{\gamma}$ capture antibody (BD ELISPOT Set, USA) and incubated overnight at $4^{\circ} \mathrm{C}$. The following day, $6 \times 10^{6}$ splenocyte suspensions from each group were stimulated with $8 \mu \mathrm{g} / \mu \mathrm{L}$ of either C (ACLVGDKVM), E1 (HSMTNAVTI), E2 (IILYYYELY) dominant single peptides $(27,28)$, or Con A (positive control), in the presence of a positive control. The plates were then incubated at $37^{\circ} \mathrm{C}$ in $5 \% \mathrm{CO}_{2}$ for $20-24 \mathrm{~h}$. The next steps were performed according to the manufacturer's instructions (BD ELISPOT Set, USA). A spot forming unit (SFU) was used to represent a T cellsecreting IFN- $\gamma$. The plates were then detected using an ELISpot plate reader (Biosys, So. Pasadena, CA).

\section{Determination of Viral Load}

To quantify the relative amount of viral RNA in the respective tissues, viral RNA was extracted from the heart, liver, spleen, lung, kidney, and brain samples using the automated Magna Pure method and a Total Nucleic Acid Kit (Roche Diagnostics), following the manufacturer's instructions. CHIKV RNA was detected with specific TaqMan probes by using one-step RT-PCR (Master RNA hybridization probes, Roche), performed on a Chromo 4 machine (Bio-Rad). The primers and probes used for CHIKV RNA quantification were CHIKV-forw AAGCTCCGCGTCCTTTA CCAAG; CHIKV-rev CCAAATTGTCCTGGTCTTCCT; and Probe: Fam-CCAATGTCTTCAGCCTGGACACCTTT-BHQ1 (29). For absolute quantification, standard curves were generated using 10-fold dilutions of CHIKV Ross RNA templates of known concentration (tittered in Vero cells by $\mathrm{TCID}_{50}$ ). Based on repeated standard curves, the formula was obtained: $y=-3.641 x+31.76$ ( $y$ means CT, $x$ means index). The extracted viral RNA from tissues were used to obtain the CT number using qRT-PCR. Weight of each tissue was measured prior to extraction. From these known data, the results were calculated and expressed as $\mathrm{TCID}_{50}$ per gram of tissue (30).

\section{Statistical Analysis}

The treatment groups were compared by two-way analysis of variance (ANOVA) and Tukey multiple comparison tests (31). Data are shown as the mean \pm the standard error of the mean (SEM). Additional data were analyzed using GraphPad Prism version 7 (GraphPad Software LLC). A P value of $<0.05$ was considered significant.

\section{RESULTS}

\section{Characterization of DNA and VLP Vaccines}

To verify the expression of the CHIKV structural polyprotein, a western blot and an indirect immune fluorescence assay of
pVRC-CHIKV transfected cells were performed using rabbit anti-CHIKV Capsid (C) and E2 antibodies, which demonstrated the expression of the capsid protein and envelope E2, as expected (Figures 1B, C). No expression was detected in mock-transfected cells.

CHIK VLPs were produced by pVRC-CHIKV transfected 293 T cells and purified by $20-60 \%$ discontinuous sucrose density gradients (Figure 2A). The purified particles were analyzed by SDS-PAGE, in which E1 and E2 glycoproteins migrated together and formed a single band at $\sim 47.3 \mathrm{kDa}$, while the precursor E3E2, was observed as a higher molecular weight band at approximately $\sim 54.6 \mathrm{kDa}$. The capsid protein formed a distinct band at $\sim 36 \mathrm{kDa}$ (Figure 2B). Electron microscopy was used to further examine the formation of CHIK VLPs. The observed VLPs had diameters of about $\sim 65-70 \mathrm{~nm}$, showing morphological characteristics similar to those of standard CHIK VLPs (Figure 2C) $(7,16)$.

\section{Co-Immunization With DNA and VLP Elicits Highest Humoral Immunity}

In this study, we tested whether co-immunization with CHIKV VLP and DNA vaccines would increase the immunogenicity of either VLP or DNA immunization alone, in mice. It was found that co-immunization using DNA and VLP vaccines induced significantly stronger VLP-, E1-, and E2- specific antibody responses, in comparison to DNA or VLP immunization alone ( $\mathrm{p}<0.001$ or $\mathrm{p}<0.0001$, respectively) (Figures 4A-C). Mice in the DNA\&VLP group clearly produced robust VLP- and E1-specific antibodies after the first injection $(p<0.001)$, which was higher than those of mice in the DNA or VLP only group $(\mathrm{p}<0.0 .001)$. When comparing the specific titers of mice against E2 proteins in each immunized group, it was found that the highest levels of anti-E2 antibodies were observed in the DNA\&VLP group, after boost immunization, followed by the VLP only and DNA only groups (Figure 4B). From the previous study results, we know that IgG1 and IgG2c isotypes were the main IgG subclasses induced after DNA or VLP immunization $(7,17)$. To further characterize the immune response generated, VLPspecific antibody subtypes were characterized. Coimmunization with DNA and VLP could induce both IgG1 and IgG2c antibodies, with significantly higher IgG1 antibody levels in comparison to IgG2c antibody levels, while the DNA or VLP only immunization groups mainly induced IgG2c antibodies (Figure 4B).

Neutralizing antibodies play an important role in the process of CHIKV infection and viral clearance. In comparison to the mock group, all immunized groups were observed to induce neutralizing antibodies in mice after the booster injection, which could cross-neutralize the heterologous CHIKV Ross strain. Among them, the DNA or VLP only immunized group induced lower neutralizing antibody titers, while co-immunization significantly increased neutralizing antibody titers (Figures 4C, D). Taken together, co-immunization induced higher levels of humoral immunity (IgG and neutralizing antibodies) than either DNA or VLP immunization alone. 
A

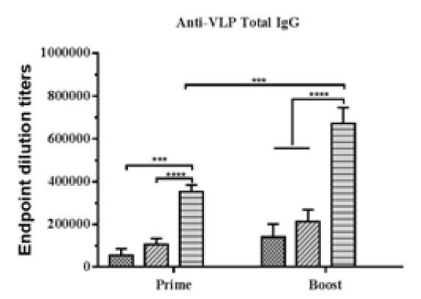

B

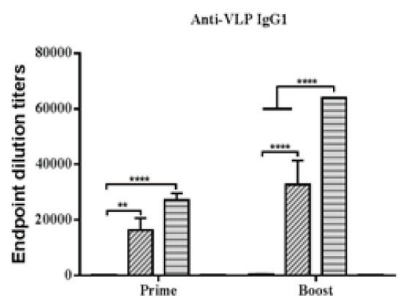

C

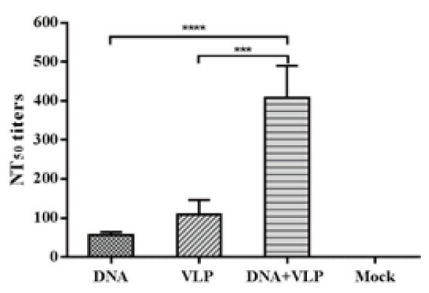

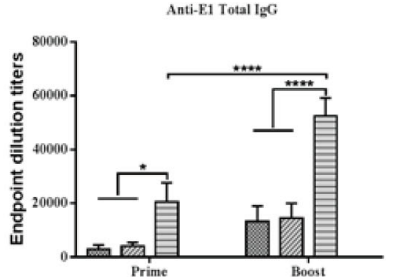
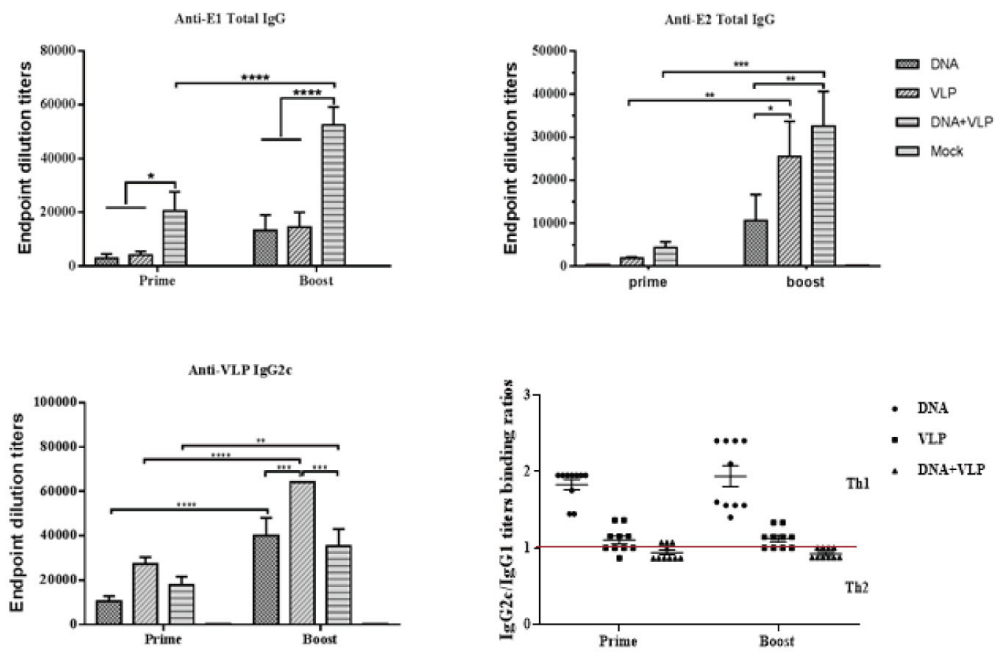

D

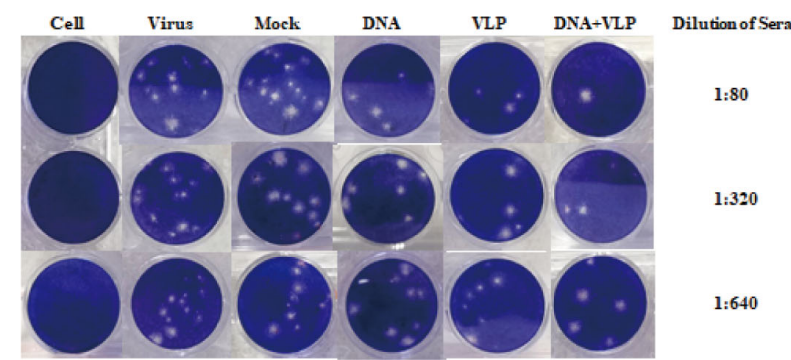

FIGURE 4 | Co-immunization-induced broad CHIKV antigen-specific IgG antibody responses and high cross-neutralization antibody response in mice. Anti CHIKV VLP, E1 and E2 antibody measurement by ELISA. Sera were collected at day 14 and 35 after the prime and boost immunization and total lgG produced was measured in each group (A). IgG subtype ELISA analysis. The graph shows VLP specific lgG1 and lgG2C antibody levels in mice, lgG2C/lgG1 binding ratios are shown for mice immunized with DNA, VLP or DNA+VLP (B). Neutralizing antibody titers against CHIKV Ross were determined by plaque reduction neutralization assays at day 35 (C). Representative results of the plaque reduction neutralization (PRNT) assay for the detection of neutralization activity in the sera of mice (D). Approximately 10-15 pfu of the virus stock (CHIKV Ross) was used to infect Vero cells in 12-well plates with heat-inactivated sera from immunized mice 14 days after the boost immunization. PRNT 50 was calculated after the plaques were counted. Data are from one experiment of three independent experiments and presented as the mean \pm SEM $(n=10)$. The statistical analysis among groups analyzed by two-way analysis of variance (ANOVA) and Tukey multiple comparison tests, ${ }^{\star \star \star *} \mathrm{p}<0.0001,{ }^{\star \star *} \mathrm{p}<0.001,{ }^{\star \star} \mathrm{p}<0.01,{ }^{\star} \mathrm{p}<0.05$.

\section{Co-Immunization With DNA and VLP Suppresses T Cell-Mediated Inflammation Responses in Mice}

To better understand immunity induced by different immunization protocols, we determined the induction of IFN- $\gamma$-producing C-, E1-, and E2-specific $T$ cells in the spleens of mice after the vaccination. Upon in vitro stimulation of spleen cells with C, E1, and E2peptides, we measured IFN- $\gamma$-producing cell spots to evaluate the $\mathrm{T}$ cell response. We found that the DNA-immunized group induced the highest level of IFN- $\gamma$ secretion after immunization. Coimmunization did not increase the induction of IFN- $\gamma$ secretion which was found to be lower than that of either the DNA or VLP only groups (Figure 5). These results confirmed that the combination of DNA and VLP induced robust Th2-polarized VLP-specific immunity and suppressed $\mathrm{T}$ cell-mediated immunity in mice.

\section{Co-Immunization Can Protect Mice From Lethal CHIKV Threat}

To determine whether there was enhanced protection against CHIKV threat in DNA and VLP co-immunized mice, in comparison to groups immunized with DNA or VLP alone, immunized mice were challenged with $1.7 \times 10^{6} \mathrm{TCID}_{50} \mathrm{CHIKV}$ Ross through intranasal infection 3 weeks after boost immunization. Body weight, survival rate, tissue viral load, and histopathological changes were evaluated (Figure 6). We found that none of the vaccinated mice suffered from disease or death (Figure 6B), and their body weight did not significantly decrease (Figure 6A). In contrast, mice in the control group started to gradually lose weight 3 days after challenge (Figure 6A), with hind limb paralysis or death occurring by the $6^{\text {th }}$ day, and all control mice died by the $9^{\text {th }}$ day (Figure 6B). Tissue viral load analysis revealed no viral nucleic acid traces in the heart, liver, spleen, and kidney tissues of the immunized groups. Only very 

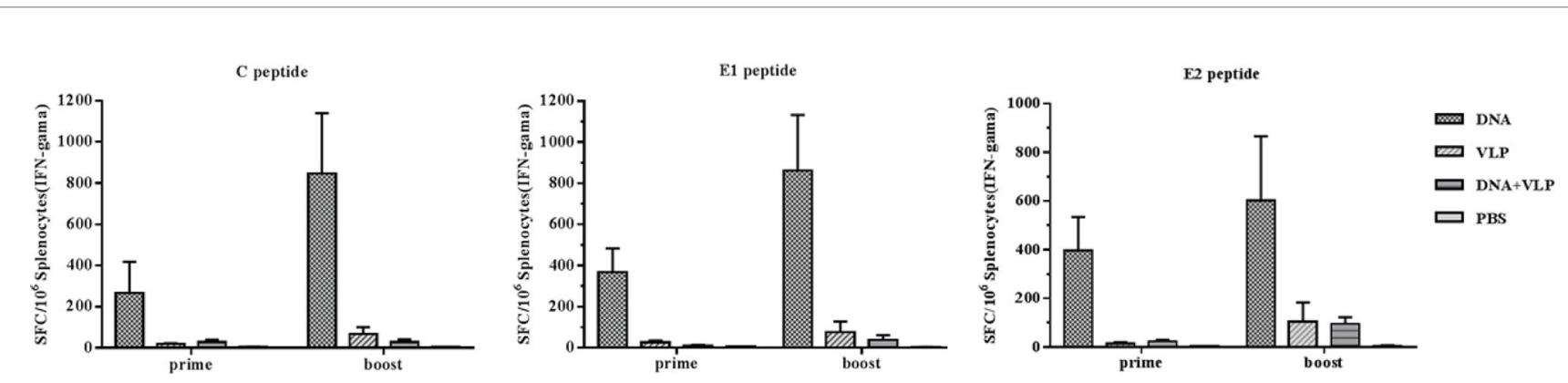

FIGURE 5 | Enzyme-linked immunospot assays of IFN- $\gamma$ secretion in immunized mice. Splenocytes were isolated from mice ( $\mathrm{n}=4)$ and stimulated with CHIKV C, E1, and E2 peptides at day 14 and 35 after the prime and boost immunization. Splenocytes secreting IFN- $\gamma$ were quantified using ELISpot assays. The data represent the mean \pm standard error [SEM], with units of SFCs per million splenocytes.

A

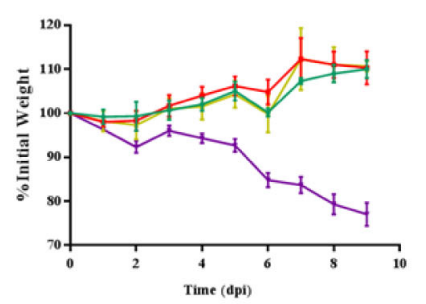

B

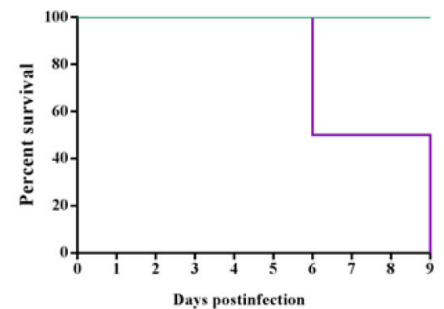

C

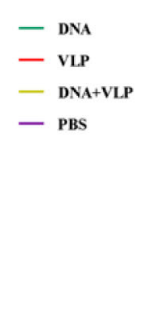

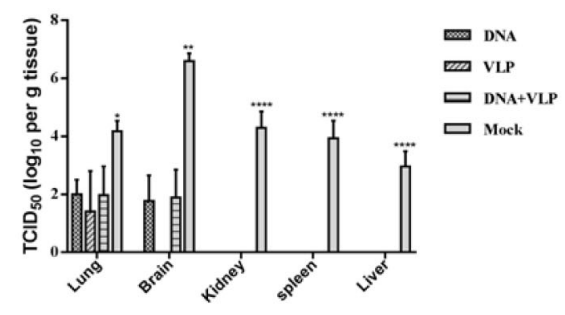

E

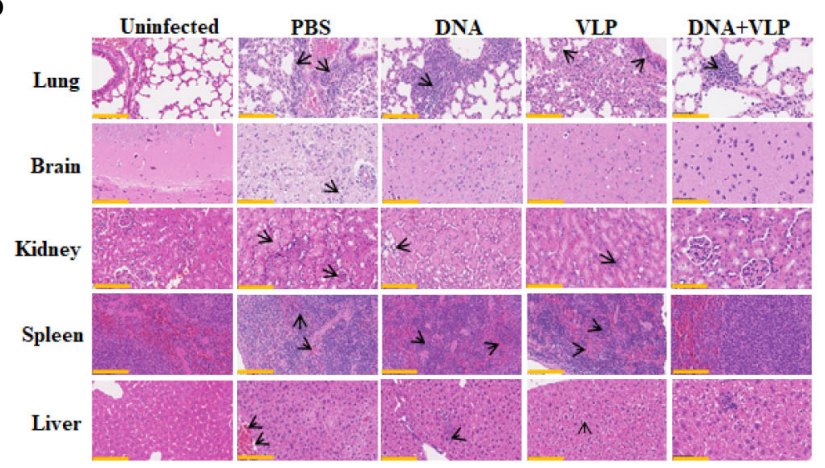

\begin{tabular}{|c|c|c|c|c|c|c|}
\hline \multirow{2}{*}{ Group } & \multicolumn{5}{|c|}{ INHAND score } & \multirow{2}{*}{ Total } \\
\hline & Lung & Brain & Kidney & Spleen & Liver & \\
\hline Uninfected & 0 & 0 & 0 & 0 & 0 & 0 \\
\hline Mock & 3 & 3 & 3 & 2 & 3 & 14 \\
\hline DNA & 3 & 0 & 3 & 2 & 1 & 9 \\
\hline VLP & 3 & 0 & 3 & 2 & 1 & 9 \\
\hline DNA+VLP & 2 & 0 & 0 & 0 & 0 & 2 \\
\hline
\end{tabular}

FIGURE 6 | Co-immunization protects mice from lethal CHIKV challenge. Mice infected with $1.7 \times 10^{7} \mathrm{TCID}_{50}$ of the CHIKV Ross were monitored daily for body weight (A) and survival (mean \pm standard error [SEM], $n=6)(\mathbf{B})$. Mice $(n=3)$ sacrificed at day 9 post challenge. The heart, liver, spleen, lung, kidney, brain, and hind limbs of mice were harvested after sacrificed. Tissue supernatants were analyzed for viral load by real-time qRT-PCR. Data represent three independent experiments and are shown as the mean \pm standard error [SEM]. The statistical analysis among groups analyzed by two-way ANOVA, ${ }^{\star \star * *} p<0.0001,{ }^{* \star} p<0.01,{ }^{*} p<0.05$. None viral load detected in heart and hind limbs of each group (C). Histopathological analysis of tissues at day 9 after challenge. Section of different tissues were stained with hematoxylin and eosin. Scale bar, $100 \mu \mathrm{m}$. The black arrowhead stood for lesions in tissue (D) (3 mice per group and 1 section per tissue). INHAND scores of challenged mice organs, on a severity scale of 0-3 (none, mild, moderate, and severe) (E).

low levels of viral RNA were detected in the lung and brain of the immunized groups, in comparison to the mock group (Figure 6C).

We performed a histological examination of mouse tissue obtained on the $9^{\text {th }}$ day, and the results of H\&E staining are shown in Figure 6D. In lung tissue sections, it was found that mock and DNA-or VLP-immunized mice exhibited a widened alveolar septum, infiltrated blood vessels, bronchial inflammatory reaction, trocar formed around blood vessels, and a homogeneous powder was found in the bronchial and bronchiolar cavities. In contrast, mice in the DNA\&VLP group showed only mild local alveolar space widening and a small amount of inflammatory cell infiltration (Figure 6D). Although viral nucleic acid was detected in the brain tissue of immunized mice, no obvious lesions were observed in brain tissue sections (Figure 6D). We observed severe lesions in kidney tissue, and minor lesions in spleen and liver tissue, of mice vaccinated with either DNA or VLP, while co-immunized mice did not show 
obvious lesions in kidney, spleen, and liver tissue sections. INHAND score analysis showed that the mock group had 14 points, the DNA or VLP group had 9 points, and the coimmunized group had only 2 points, which was significantly lower in comparison to that of the other groups (Figure 6E). Taken together, DNA and VLP co-immunization provided the best protection after CHIKV Ross challenge.

\section{DISCUSSION}

In this study, we developed novel CHIKV DNA and VLP vaccines, and evaluated their immunological and protective effects in mice, using different vaccination protocols. The results showed that all vaccination protocols were effective in protecting mice from lethal CHIKV challenge. Notably, co-immunization of mice with DNA and VLP vaccines exhibited the mildest histopathological changes and lowest INHAND scores, in comparison to mice with either DNA or VLP vaccination alone. This promising protection observed in mice co-immunized with DNA and VLP vaccines may be associated with higher levels of humoral immunity (IgG and neutralizing antibodies).

In a previous study, a DNA vaccine expressing E1-E2-C CHIKV proteins mainly induced IgG1 subtype antibodies, while a VLP vaccine induced a balanced $\operatorname{IgG} 1 / \operatorname{IgG} 2 \mathrm{c}$ antibody response after immunization (17); however, IgG2c subtype antibodies were mainly induced in our immunized groups. The difference in vaccine outcomes between this previous report and the present study may have resulted from differences in the target antigens (the CHIKV full structure gene was used in this study) and vaccination protocols were used. Of note, natural CHIKV infection is dominated by $\operatorname{IgG} 2 \mathrm{c}$ (32). Furthermore, we found that the DNA\&VLP group induced specific IgG antibodies that recognized VLP, E1, and E2, in mice after the first immunization, and the level of IgG antibodies significantly increased after booster immunization. Co-immunization can also induce high levels of IgG1 and IgG2c antibodies against VLP, in which the Th2 response, represented by the presence of IgG1 antibody, dominates. It has been found that mixing polypeptides, proteins, inactivated viruses, or VLPs with DNA can synergistically enhance humoral immune response, improve neutralizing antibody levels, and produce a lasting immune protective effect (33-35).

Here, PRNT assays were applied to evaluate the crossneutralizing ability of immunized sera to CHIKV Ross. It was found that co-immunization induced the highest level of crossneutralizing antibodies, which was significantly higher in comparison to mice treated with either DNA or VLP vaccines alone. This was consistent with a previous report where an HIV/ SIV DNA vaccine, combined with protein in a co-immunization protocol, elicited the highest humoral responses to the viral envelope in mice and macaques (36). In addition, previous respiratory syncytial virus (RSV) vaccine studies have shown that adding DNA to VLP to immunize mice, could provide longterm protection against RSV infection, without the risk of lung disease (20).
In 2003, Wang et al. found that immunization with either a DNA vaccine or a protein vaccine could activate T cells, while the combination of DNA and protein inhibited T cell immunity (37). In combined HIV DNA and protein immunization protocols, the cellular immune response was enhanced, which led to an increase in IFN- $\gamma$, TNF- $\alpha$ and multi-functionality among $\mathrm{CD}^{+} \mathrm{T}$ cells and dominance of Th1-polarized Ab-specific antibodies (36). While DNA and protein combined immunization in Alzheimer's disease induced robust Th2polarized Ab-specific antibodies, suppressed or eliminated unwanted adverse $\mathrm{T}$ cell-mediated immune responses. At the same time, co-immunization reduced the production of inflammatory cytokine IFN- $\gamma$ and increased the production of IL- 4 in CD ${ }^{+} \mathrm{T}$ cells, suggesting that an anti-inflammatory effect was induced in the co-immunized mice (38). Here, we found that the cellular immune response, upon treatment with individual VLP or DNA vaccines, was stronger than that induced by coimmunization with CHIKV DNA and VLP. In addition, the combination of VLP and DNA failed to enhance the cellular immune response. Therefore, we conclude that co-immunization of CHIKV VLP with DNA vaccines inhibits cellular immunity, which is otherwise increased by either DNA vaccination alone.

When mice were challenged with a lethal dose of CHIKV Ross, we found that all immunized mice did not develop the disease or die. Further analysis of histopathological sections revealed that the mock group, as well as the VLP, or DNA immunization groups had significant lung lesions, while mice in the DNA\&VLP group had only mild lung lesions. This may be related to the nasal inoculation of CHIKV Ross. CHIKV Ross exhibited a certain neurotoxicity (39). Previous studies have shown that infection with CHIKV Ross can damage brain tissue in mice (40). Here, only the mock group had significant brain lesions, while the brain tissues of immunized mice were normal. However, it was found that different degrees of pathological damage were observed in the kidney, spleen, and liver of mice with either VLP or DNA immunization, while co-immunization did not reveal any pathological changes in these tissues. We suggest that coimmunization has a better protective effect as compared to treatment with DNA or VLP vaccines alone, especially with respect to the production of neutralizing antibodies, which play a vital role in the process of CHIKV infection and viral clearance (41-43). Whether these differences in pathology due to humoral response are more effective in protection against $\mathrm{CHIKV}$ infection than the $\mathrm{T}$ cell mediated response remains to be elucidated. From our results, we found that the $\mathrm{T}$ cell-mediated response was significantly higher in the DNA only group than in the coimmunization group, but it did not provide better protection than co-immunization. No significant difference was found between the VLP only group and the co-immunization group $\mathrm{T}$ cell-mediated response, while co-immunization provided better protection than the VLP only group. This may be because coimmunization induced higher levels of humoral response than VLP or DNA immunization. We found that the humoral response is more effective in protection against CHIKV infection than the $\mathrm{T}$ cell-mediated response. Therefore, future CHIKV vaccines should focus on generating protective antibodies. 
Several limitations of the current study need to be examined. $\mathrm{CHIKV}$ is an arthritogenic virus. Future studies will explore whether the DNA\&VLP vaccine strategy could be an excellent strategy to reduce the joint footpad swelling and muscle pathology in the well-defined footpad model. Second, in the DNA only group, a T-cell mediated response was observed, which is very likely a Th1 response (high IgG2c, low IgG1). Additional studies should identify the role of these T cells. Lastly, we only observed the DNA\&VLP vaccine strategy in C57BL/6 mice. Future studies should upgrade the vaccine production and explore protection efficacy and related mechanisms in larger animals.

In summary, our data revealed that co-immunization with DNA and VLP vaccines did not enhance the cellular immune response in mice, but significantly enhanced humoral immunity and induced higher levels of antibodies with cross-neutralizing activity, in comparison to treatment with either component alone. After CHIKV Ross challenge, co-immunization provided greater cross-protection in mice. We concluded that the VLP and DNA co-immunization method developed here, might be a very promising strategy to improve immunity and protection against CHIKV infection.

\section{DATA AVAILABILITY STATEMENT}

The original contributions presented in the study are included in the article/Supplementary Material, further inquiries can be directed to the corresponding author.

\section{REFERENCES}

1. Powers AM. Vaccine and therapeutic options to control chikungunya virus. Clin Microbiol Rev (2018) 31:1-29. doi: 10.1128/CMR.00104-16

2. Reyes-Sandoval A. 51 years in of Chikungunya clinical vaccine development: A historical perspective. Hum Vaccines Immunother (2019) 15:2351-8. doi: 10.1080/21645515.2019.1574149

3. Erasmus JH, Rossi SL, Weaver SC. Development of Vaccines for Chikungunya Fever. J Infect Dis (2016) 214:S488-96. doi: 10.1093/infdis/jiw271

4. Ramphan S, Khongwichit S, Saisawang C, Kovanich D, Ketterman AJ, Ubol S, et al. Ubiquitin-Conjugating Enzyme E2 L3 is Downregulated by the Chikungunya Virus nsP2 Protease. Proteomics - Clin Appl (2018) 12:1-33. doi: $10.1002 /$ prca.201700020

5. Sanchez-San Martin C, Nanda S, Zheng Y, Fields W, Kielian M. CrossInhibition of Chikungunya Virus Fusion and Infection by Alphavirus E1 Domain III Proteins. J Virol (2013) 87:7680-7. doi: 10.1128/jvi.00814-13

6. Weger-Lucarelli J, Aliota MT, Wlodarchak N, Kamlangdee A, Swanson R, Osorio JE. Dissecting the Role of E2 Protein Domains in Alphavirus Pathogenicity. J Virol (2016) 90:2418-33. doi: 10.1128/jvi.02792-15

7. Metz SW, Gardner J, Geertsema C, Le TT, Goh L, Vlak JM, et al. Effective Chikungunya Virus-like Particle Vaccine Produced in Insect Cells. PloS Negl Trop Dis (2013) 7:e2124. doi: 10.1371/journal.pntd.0002124

8. Li L, Jose J, Xiang Y, Kuhn RJ, Rossmann MG. Structural changes of envelope proteins during alphavirus fusion. Nature (2010) 468:705-8. doi: 10.1038/ nature09546

9. Gao S, Song S, Zhang L. Recent Progress in Vaccine Development Against Chikungunya Virus. Front Microbiol (2019) 10:2881. doi: 10.3389/ fmicb.2019.02881

10. Ali MG, Zhang Z, Gao Q, Pan M, Rowan EG, Zhang J. Recent advances in therapeutic applications of neutralizing antibodies for virus infections : an overview. Immunol Res (2020) 68:325-39. doi: 10.1007/s12026-020-09159-Z

11. Chang LJ, Dowd KA, Mendoza FH, Saunders JG, Sitar S, Plummer SH, et al. Safety and tolerability of chikungunya virus-like particle vaccine in healthy

\section{ETHICS STATEMENT}

The animal study was reviewed and approved by Committee on the Ethics of Animal Experiments of the Chinese Center for Disease Control and Prevention.

\section{AUTHOR CONTRIBUTIONS}

Conceptualization: WT and ZZ. Formal analysis: ZZ, PN, and YD. Investigation: ZZ, JS, YPD, and WW. Resources: LZ and PZ. Writing-original draft preparation: ZZ and WT. Writingreview and editing: ZZ, LZ, and WT. Project administration: $\mathrm{BH}, \mathrm{WLW}$, and WT. All authors contributed to the article and approved the submitted version.

\section{FUNDING}

This work was supported by the National Key Research and Development Program of China (2018YFC1200602 and 2016YFD0500301) and the National Major Project for Control and Prevention of Infectious Disease in China (2016ZX10004001-003 and 2018ZX10731-101). The funding agencies had no role in the study design, data collection, data analysis, decision to publish, or preparation of the manuscript.

adults: A phase 1 dose-escalation trial. Lancet (2014) 384:2046-52. doi: 10.1016/S0140-6736(14)61185-5

12. Reisinger EC, Tschismarov R, Beubler E, Wiedermann U, Firbas C, Loebermann $\mathrm{M}$, et al. Immunogenicity, safety, and tolerability of the measles-vectored chikungunya virus vaccine MV-CHIK: a double-blind, randomised, placebo-controlled and active-controlled phase 2 trial. Lancet (2018) 392:2718-27. doi: 10.1016/S0140-6736(18)32488-7

13. Lum FM, Couderc T, Chia BS, Ong RY, Her Z, Chow A, et al. Antibodymediated enhancement aggravates chikungunya virus infection and disease severity. Sci Rep (2018) 8:1-14. doi: 10.1038/s41598-018-20305-4

14. Hallengard D, Lum F-M, Kummerer BM, Lulla A, Lulla V, Garcia-Arriaza J, et al. Prime-Boost Immunization Strategies against Chikungunya Virus. J Virol (2014) 88:13333-43. doi: 10.1128/jvi.01926-14

15. Abeyratne E, Freitas JR, Zaid A, Mahalingam S, Taylor A. Attenuation and Stability of CHIKV-NoLS, a Live-Attenuated Chikungunya Virus Vaccine Candidate. Vaccines (2019) 7:2. doi: 10.3390/vaccines7010002

16. Saraswat S, Athmaram TN, Parida M, Agarwal A, Saha A, Dash PK. Expression and Characterization of Yeast Derived Chikungunya Virus Like Particles (CHIK-VLPs) and Its Evaluation as a Potential Vaccine Candidate. PloS Negl Trop Dis (2016) 10:1-19. doi: 10.1371/journal.pntd.0004782

17. Bao H, Ramanathan AA, Kawalakar O, Sundaram SG, Tingey C, Bian CB, et al. Nonstructural protein 2 (nsP2) of chikungunya virus (CHIKV) enhances protective immunity mediated by a CHIKV envelope protein expressing DNA vaccine. Viral Immunol (2013) 26:75-83. doi: 10.1089/vim.2012.0061

18. Li J, Jin H, Zhang A, Wang B, Zhang F. Enhanced contraceptive response by co-immunization of DNA and protein vaccines encoding the mouse zona pellucida 3 with minimal oophoritis in mouse ovary. J Gene Med (2012) 9:1095-103. doi: 10.1002/jgm

19. Ishikawa T, Takasaki T, Kurane I, Nukuzuma S. Co-immunization with West Nile DNA and inactivated vaccines provides synergistic increases in their immunogenicities in mice. Microbes Infect (2007) 9:1089-95. doi: 10.1016/j.micinf.2007.05.013

20. Hwang HS, Kwon YM, Lee JS, Yoo SE, Lee YN, Ko EJ, et al. Co-immunization with virus-like particle and DNA vaccines induces protection against 
respiratory syncytial virus infection and bronchiolitis. Antiviral Res (2014) 110:115-23. doi: 10.1016/j.antiviral.2014.07.016

21. Mainou BA, Zamora PF, Ashbrook AW, Dorset DC, Kim KS, Dermody TS. Reovirus cell entry requires functional microtubules. MBio (2013) 4:1-10. doi: 10.1128/mBio.00405-13

22. Zhang N, Zhao H, Leiliang Z. Fatty Acid Synthase Promotes the Palmitoylation of Chikungunya Virus nsP1. J Virol (2019) 93:1-12. doi: 10.1128/JVI.01747-18

23. Luo Z, Xu Z, Tang H, Peng H, Zhao L, Qi Z, et al. Construction and identification of Chikungunya virus infectious clones. Int J Viro (2018) 26:337-40. doi: 10.3760/cma.j.issn.1673-4092.2019.05.013

24. Barouch DH, Yang Z, Kong W, Korioth-schmitz B, Sumida SM, Truitt DM, et al. A Human T-Cell Leukemia Virus Type 1 Regulatory Element Enhances the Immunogenicity of Human Immunodeficiency Virus Type 1 DNA Vaccines in Mice and Nonhuman Primates. J Virol (2005) 79:8828-34. doi: 10.1128/JVI.79.14.8828

25. Azila N, Azami M, Moi ML, Takasaki T. Chapter 25 Neutralization Assay for Chikungunya Virus Infection : Plaque Reduction Neutralization Test. Methods Mol Biol (2016) 1426:273-82. doi: 10.1007/978-1-4939-3618-2

26. Zhang Y-N, Deng C-L, Li J-Q, Li N, Zhang Q-Y, Ye H-Q, et al. Infectious Chikungunya Virus (CHIKV) with a Complete Capsid Deletion: a New Approach for a CHIKV Vaccine. J Virol (2019) 9393:e00504-19. doi: $10.1128 /$ jvi.00504-19

27. Zhan Y, Deng Y, Huang B, Song Q, Wang W, Yang Y, et al. Humoral and cellular immunity against both ZIKV and poxvirus is elicited by a twodose regimen using DNA and non-replicating vaccinia virus-based vaccine candidates. Vaccine (2019) 37:2122-30. doi: 10.1016/j.vaccine. 2019.02.063

28. Muthumani K, Lankaraman KM, Laddy DJ, Sundaram SG, Chung CW, Sako E, et al. Immunogenicity of novel consensus-based DNA vaccines against Chikungunya virus. Vaccine (2008) 26:5128-34. doi: 10.1016/ j.vaccine.2008.03.060

29. Garcia-Arriaza J, Cepeda V, Hallengard D, Sorzano COS, Kummerer BM, Liljestrom P. Esteban M. A Novel Poxvirus-Based Vaccine, MVA-CHIKV, Is Highly Immunogenic and Protects Mice against Chikungunya Infection. J Virol (2014) 88:3527-47. doi: 10.1128/jvi.03418-13

30. Sanchez-Felipe L, Vercruysse T, Sharma S, Ma J, Lemmens V, Van Looveren $\mathrm{D}$, et al. A single-dose live- attenuated YF17D-vectored SARS-CoV-2 vaccine candidate. Nature (2020) 590:320-5. doi: 10.1038/s41586-020-3035-9

31. Metz SW, Martina BE, van den Doel P, Geertsema C, Osterhaus AD, Vlak JM, et al. Chikungunya virus-like particles are more immunogenic in a lethal AG129 mouse model compared to glycoprotein E1 or E2 subunits. Vaccine (2013) 31:6092-6. doi: 10.1016/j.vaccine.2013.09.045

32. Arévalo MT, Huang Y, Jones CA, Ross TM. Vaccination with a chikungunya virus-like particle vaccine exacerbates disease in aged mice. PloS Negl Trop Dis (2019) 13:1-18. doi: 10.1371/journal.pntd.0007316

33. Gardner J, Anraku I, Le TT, Larcher T, Major L, Roques P, et al. Chikungunya Virus Arthritis in Adult Wild-Type Mice. J Virol (2010) 84:8021-32. doi: $10.1128 /$ jvi.02603-09
34. You SH, Kim T, Choi JH, Park G, Lee KN, Kim B, et al. Coinjection of a vaccine and anti-viral agents can provide fast-acting protection from footand-mouth disease. Antiviral Res (2017) 143:195-204. doi: 10.1016/ j.antiviral.2017.04.009

35. Min D, Jian-zhong Z. SSB peptide and DNA co-immunization induces inhibition of anti-dsDNA antibody production in rabbits. Chin Med J (Engl) (2008) 121:227-30. doi: 10.1097/00029330-200802010-00008

36. Li J, Valentin A, Kulkarni V, Rosati M, Kelly R, Alicea C, et al. HIV / SIV DNA vaccine combined with protein in a co-immunization protocol elicits highest humoral responses to envelope in mice and macaques. Vaccine (2013) 31:3747-55. doi: 10.1016/j.vaccine.2013.04.037

37. Li J, Geng S, Xie X, Liu H, Zheng G, Sun X, et al. Caveolin-1-Mediated Negative Signaling Plays a Critical Role in the Induction of Regulatory Dendritic Cells by DNA and Protein Coimmunization. J Immunol (2012) 189:2852-9. doi: 10.4049/jimmunol.1102828

38. Liu S, Shi D, Wang HC, Yu YZ, Xu Q, Sun ZW. Co-immunization with DNA and protein mixture: A safe and efficacious immunotherapeutic strategy for Alzheimer's disease in PDAPP mice. Sci Rep (2015) 5:1-9. doi: 10.1038/ srep07771

39. Abdelnabi R, Delang L, Neyts J. Antiviral drug discovery against arthritogenic alphaviruses : Tools and molecular targets. Biochem Pharmacol (2020) 174:113777. doi: 10.1016/j.bcp.2019.113777

40. Wang E, Volkova E, Adams AP, Forrester N, Xiao SY, Frolov I, et al. Chimeric alphavirus vaccine candidates for chikungunya. Vaccine (2008) 26:5030-9. doi: 10.1016/j.vaccine.2008.07.054

41. Mallilankaraman K, Shedlock DJ, Bao H, Kawalekar OU, Fagone P, Ramanathan AA, et al. A DNA vaccine against Chikungunya virus is protective in mice and induces neutralizing antibodies in mice and nonhuman primates. PloS Negl Trop Dis (2011) 55:e928. doi: 10.1371/ journal.pntd.0000928

42. Muthumani K, Block P, Flingai S, Muruganantham N, Chaaithanya IK, Tingey C, et al. Rapid and Long-Term Immunity Elicited by DNA-Encoded Antibody Prophylaxis and DNA Vaccination Against Chikungunya Virus. J Infect Dis (2016) 214:369-78. doi: 10.1093/infdis/jiw111

43. Couderc T, Khandoudi N, Grandadam M, Visse C, Gangneux N, Bagot S, et al. Prophylaxis and therapy for chikungunya virus infection. J Infect Dis (2009) 200:516-23. doi: $10.1086 / 600381$

Conflict of Interest: The authors declare that the research was conducted in the absence of any commercial or financial relationships that could be construed as a potential conflict of interest.

Copyright (๑ 2021 Zhao, Deng, Niu, Song, Wang, Du, Huang, Wang, Zhang, Zhao and Tan. This is an open-access article distributed under the terms of the Creative Commons Attribution License (CC BY). The use, distribution or reproduction in other forums is permitted, provided the original author(s) and the copyright owner(s) are credited and that the original publication in this journal is cited, in accordance with accepted academic practice. No use, distribution or reproduction is permitted which does not comply with these terms. 